\title{
Karl Wolfskehls Gedichtzyklus Mittelmeer oder die Fünf Fenster Ein Zahlenspiel um Stefan George?
}

Karl Wolfskehls Zyklus Mittelmeer oder die Fünf Fenster gehört als erster Zyklus, nach dem "Präludium", zu den insgesamt sechs Teilen des Großzyklus Die Drei Welten und das Lebenslied. Wolfskehl sah in diesem im neuseeländischen Exil geschriebenen Großzyklus Höhepunkt und Summe seines Schaffens. Dies ist auch generell so gewürdigt worden. Besonders der Hiob-Zyklus, das »Lebenslied « An die Deutschen, oder als Einzeltext, der dann im Mittelmeer-Zyklus Aufnahme fand, das berühmte Feigenbaum-Gedicht, haben ihre Interpreten und Liebhaber gewonnen. Vom Mittelmeer-Zyklus als ganzem kann man das kaum sagen: wenig ist bisher darüber geschrieben worden, und das wenige ist dann einem anderen Zusammenhang untergeordnet.

Dies mag daran liegen, daß man diesen Zyklus weitgehend als sperrig und unausgewogen, ja als den am wenigsten gelungenen Teil der Drei Welten ansieht. Wolfskehl selbst macht es deutlich, daß es mit seinem "Mare Nostrum", wie er das Gebilde immer wieder nennt, eine Besonderheit hat: In Briefen hüllte er den Zyklus in eine seltsame Zahlenmystik und verband ihn derart weihevoll mit Stefan George, daß Nicht-Georgianer eher mißtrauisch werden mußten. Dieses Mißtrauen muß Wolfskehl geahnt haben, oder vielleicht wollte er sich nur den Eingeweihten mitteilen, denn, im Gegensatz zu den anderen Texten des Großzyklus, bei denen er für weitere Verbreitung sorgte, behielt er die dem "Mare Nostrum" zugeordneten Texte nur wenigen, dem George-Kreis verbundenen Empfängern vor.

Dem Zahlenspiel und der George-Nähe soll hier nachgegangen werden: als Vorarbeit zu einer umfangreichen Interpretation und Kommentierung, die noch zu leisten wäre. Als Einstieg sei vergleichend ein kleiner Text gewählt, der wie kein zweiter, durch gedankliche Nähe aber ästhetische Ferne, geeignet ist, die Besonderheiten Wolfskehls zu beleuchten.

1929 erschien in Neuseeland ein schmaler Gedichtband mit dem Titel From a Garden in the Antipodes. Verfasserin war die neuseeländische Lyrikerin Ursula Bethell, eine gebürtige Engländerin aus wohlhabender Familie, die in Oxford und in der Schweiz zur Schu- 
le gegangen war, fließend Deutsch sprach, und in Dresden Musik studiert hatte. Ihr späteres Leben in Christchurch empfand sie oft als eine Art Exil, und ihre Gedichte, wie schon der Titel des genannten Bandes andeutet, leben aus der Spannung zwischen der geistigen Heimat einerseits und der physischen Heimat andererseits: "Emerging into Christchurch streets the barbarity struck me more acutely than ever", schreibt sie 1937 in einem Brief. ${ }^{1}$ In dem Band von 1929 findet sich das folgende kleine Gedicht mit dem unauffällig leisen Titel „Detail“:

My garage is a structure of excessive plainness,

It springs from a dry bank in the back garden,

It is made of corrugated iron,

And painted all over with brick-red.

But beside it I have planted a green Bay-tree,

A sweet Bay, an Olive, and a Turkey Fig,

A Fig, an Olive, and a Bay.2

In sieben lakonischen Zeilen wird die "barbarity" Neuseelands kontrastiert mit einer zurückgelassenen und nun herbeizitierten mittelmeerischen Welt, die sich in der sakralen, und daher groß geschriebenen, Dreizahl von Lorbeer ("Bay-tree"), Olive und Feige konkretisiert. Dies geschieht dergestalt, daß die vorletzte Zeile vom Lorbeer über die Olive zur Feige hin ansteigt, um dann in der Wiederholung rücklaufend, also chiastisch, von der Feige wieder zum Lorbeer abzufallen, mithin in der Feige kulminiert. Zweimal erscheinen Olive und Feige, dreimal aber der Lorbeer, wie ein rahmender Kranz um Anfang und Ende der Reihung gelegt. Wenn damit auf den Dichterruhm angespielt wird, so wird er bescheiden versteckt hinter dem Wort "Bay-tree" als alias für "Laurel".

Kunstvoll ist auch der Gebrauch, den Ursula Bethell von der Leerzeile macht. Sie läßt durch die Trennung innerhalb der Siebenzahl der Verse ein zweites Mal die Dreizahl erkennen: in der ersten Strophe die grundsolide Vier als Eckpfosten ihrer neuseeländischen Umgebung, in der zweiten die sakrale Drei als mythische Sphäre, und sie läßt die Distanz fühlbar werden, die geistige wie die geographische, die zwischen den beiden Welten liegt. Umso entschlossener kann dann, mit leisem Triumph, das "But" der Gegenwelt einsetzen, mit der hoffnungsvoll grünen und symbolträchtigen

1 Bethell, Ursula, Collected Poems, Wellington 1997, Introduction, S. XII.

2 Ebd., 1, S. 5 
Vegetation von Klassik und Mittelmeer, die das ziegelrote Wellblech der Garage und den dürren Hang verdecken sollen.

Karl Wolfskehl hätte mit dem Gedicht und seiner Autorin bekannt sein können. 1936 gab Ursula Bethell ihren zweiten Band an die Caxton Press in Christchurch, deren Leiter damals der später in Neuseeland sehr bekannte Lyriker Denis Glover war. 1941 besuchte Wolfskehl die Caxton Press und freundete sich mit Glover an: "Hier in New Zealand [sic!] selbst entwickelt sich hoffnungsvolles geistiges Leben um den Mittelpunkt der 'Caxton Press' in Christchurch", schreibt er am 31. Juli 1942 an Kurt Wolff und fährt fort: "Mit den dortigen wie mit den hier lebenden Vertretern dieses guten Beginns bin ich in Fühlung." (BaN II, S. 514)

$\mathrm{Zu}$ dieser Zeit arbeitete Wolfskehl schon an seinem Zyklus Mittelmeer oder die Fünf Fenster, der 1946, bis auf wenige Änderungen und Zusätze, abgeschlossen war. Schon 1938/39 war das bekannte Gedicht „Feigenbaum“ entstanden, das dann unter dem Titel „Exules ambo“" Teil des Zyklus wurde. Die Spannung zwischen den beiden Welten stellt sich für Wolfskehl im übrigen in ähnlichen Worten dar wie für Ursula Bethell: "Mein Zimmer ist hell, großfenstrig, mit einem Blick gen Westen, träumenderweise bis zum Mittelmeer", heißt es, wohl in Anspielung auf die "Fünf Fenster“, in einem Brief vom 1. 11. 1946 (BaN II, S. 899). Von dem "pionirischen", "primitiven Gepräge" seines Daseins spricht er im selben Jahr (BaN II, S.107), von den "Wohnkisten", in denen die Menschen leben, und der Öde von Aucklands Mitte: Auch der Begriff der "barbarity", die Ursula Bethell in den Straßen von Christchurch erschreckt hatte, erscheint bei Wolfskehl, wenn im Mittelmeer-Zyklus, in den "Tabellae“ des „Dritten Fensters“, von der Küste Aucklands gesprochen wird als von des "Exils barbarischer Klippenbai". Natürlich finden sich, wie wir wissen, auch der Lorbeer und die Olive (als "Olivet" = lat. olivetum, Olivenhain) in Wolfskehls Zyklus - der Lorbeer ebenso im Exil wie der Feigenbaum in „Exules ambo“, dem 'Ersten' der 'Fünf Fenster', durch die er sich, wie es im Brief hieß, ans Mittelmeer träumte:

\section{Laurus in Exilio}

Auch hier des Phöbus-Lieblings griechische Züge:

Der schlanke Stamm, feinästig, schmal umlaubt,

Herbstsüsse Jungheit dunstend. Doch wer trüge

Sein Haupt so hoch, dass du als Kranz erlaubt. 
Auch hier finden sich die Motive von Dichterruhm; Bekränzung samt Bescheidenheitstopos, nur ist das, was in Ursula Bethells "Baytree" nur gerade anklingt, hier voll ausgeführt, wie überhaupt beim späten Wolfskehl Pathos und dröhnende Sprache alles Leise, alles verhaltene Understatement, wie sie für die moderne Lyrik kennzeichnend sind, ersetzen.

Damit sind wir bei dem Punkt angelangt, auf den es hier ankommen soll. Nicht um die Konstruktion eines möglichen Einflusses soll und kann es gehen, sondern um die so völlig verschiedenartige Bewältigung, mit zum Teil gleicher Bildersprache, einer ähnlich gelagerten Situation - bei allem Unterschied in der Größenordnung des Leidensdrucks und des persönlichen Schicksals. Wir haben das Paradox, daß sich in dem "pionirischen" Neuseeland eine Lyrik finden läßt, die, zeitlich noch vor den späten Texten Wolfskehls liegend, in ihrer Machart dennoch ganz der literarischen Moderne verpflichtet ist, während der Europäer Wolfskehl, in dem für die Emigrantenlyrik als typisch erkannten "ästhetischen Regreß",3 sich einer Form und Sprache bedient, deren Wurzeln im Vorexpressionismus liegen. Psychologisch läßt sich das sicher so erklären, daß der deutsche Emigrant verzweifelt die Tradition, aus der er stammt, als Stütze und zur Selbstvergewisserung festzuhalten versucht, während die zur Neuseeländerin gewordene Engländerin, gefestigt in ihrer englischsprachigen Umgebung verbleibend, den Schritt nach vorne wagen kann. So bleibt für Ursula Bethell die Entfernung von der europäischen Lebens- und Geisteswelt eben nur ein Detail, Detail, und wird entsprechend lakonisch vermerkt, während dieselbe Entfernung für Wolfskehl zum grundstürzenden Existential wurde. Hinzu kommt natürlich die Tatsache, daß für Bethell diese Welt 1929 noch nicht bedroht war, während Wolfskehl ihren Untergang bezeugen muß. Schon 1938, also noch vor Ausbruch des Zweiten Weltkrieges, sieht Wolfskehl aus Verunsicherung und Bedrohung seine Aufgabe als Mensch und als Dichter im Exil erwachsen: "Und wir Europäer, nun da und dort gelandet, in immer nur notdürftig gezimmerter Seelenbehausung untergekrochen, wir müssen ausharren, es geht um die Erhaltung, die Sicherung eines vieltausendjährigen Kontinuums, das vielleicht noch nie so bedroht war, wie in unsrer Zeit; denn nicht wie in den Tagen, da die antike Welt zerbrach, kommen die Stürme von außen: der Grund selber wankt, bebt in sich [...]" (BaN II, S. 278). So konnte das

3 Trapp, Frithjof, Deutsche Literatur zwischen den Weltkriegen II. Literatur im Exil, Bern, Frankfurt am Main, New York, 1983, S. 106. 
Mittelmeer für ihn kein "Detail" bleiben, sondern mußte zur Summe werden: "Ich habe darin alles ausgesprochen, was mir über dies Ur-Mysterium der weissen Menschheit erlaubt und eröffnet ist", schreibt er 1946 an Max Brünn (BaN I, S. 292).

Allein schon der Grundgedanke des Zyklus schließt jegliche Lakonik, jegliche Untertreibung und jeglichen leisen Ton aus, denn er ist von einem hyperbolischen Anspruch: daß er, Karl Wolfskehl, der einzig noch lebende und zuletzt noch berufene Hüter jenes "vieltausendjährigen Kontinuums" sei, der "letzte Seh'r", der "[i]n letzter Sohnschaft heim in Unser Meer" "taucht", wie es bezeichnenderweise in den Schlußzeilen des letzten, des "Fünften Fensters" mit dem Titel „Ultimus Vatum" lautet. Die Vaterschaft dieses Sohnes ist natürlich die Stefan Georges. Das Wort "Sohnschaft" stammt aus dessen Stern des Bundes und meint das Berufensein in den Kreis des "Meisters", der den so Erwählten mit "Sendung" und "Segen" auszeichnet:

Durch die sendung durch den segen

Tauscht ihr sippe stand und namen

Väter mütter sind nicht mehr

Aus der sohnschaft der erlosten

Kür ich meine Herrn der Welt. ${ }^{4}$

Die zentrale Stelle für eine solche "Kür" und "Sohnschaft" sind die Eingangsverse zum "Fünften Fenster", wo Wolfskehl in ein Zwiegespräch mit George eintritt:

Den Kaisern zugehörig,

Heimlich und aufrecht, weltweis, gottestörig

Vèrblieb ich, jüdisch, römisch, deutsch zugleich,

Ein Mann des Altreichs, bis ins Neue Reich

Der Wandlung Herr mich rief: "Du ganz von eh,

Ganz Ursprung, Gestern - Jüngst im Morgen steh!

"Die Tracht und Pracht von damals bringst du mit.

Entbürde, gürte dich zum Tänzerschritt.

Vorfahr, nun Unser Sohn, liebend verspür

Was euch vertraut ist, obliegt: Sende, Kür!"

Und in der letzten der 41 ,Tabellae“ zählt Wolfskehl sich zu "den

4 George, Stefan, Werke. München und Düsseldorf 1958, S. 382. 
Erlauchten,/Die unser Meister sich befahl zum Heer,/ Zur Schutzmacht Neuem Reiche Mittelmeer". Wolfskehl wiederholt also für sich nach dem Zweiten Weltkrieg, was George nach dem Ersten Weltkrieg als die eigentliche Aufgabe benannt hatte: daß die besten des Volkes, durch Leiden geläutert, an der Schaffung eines "Neuen Reiches" arbeiten, einer Welt der geistigen Erneuerung. Speziell wird mit den Worten "Kür" und "Sende" verwiesen auf das Gedicht "Sprüche an die Toten", mit dem George nach dem Ersten Weltkrieg den inneren Verfall Deutschlands beklagt und, in der Geste des Propheten und Sehers, weiteres Unheil verkündet und zu einer Umkehr mahnt:

\title{
Wenn je dieses volk sich aus feigem erschlaffen \\ Sein selber erinnert der kür und der sende: \\ Wird sich ihm eröffnen die göttliche deutung \\ Unsagbaren grauens ...... $]^{5}$
}

In dieser Nachfolge will Wolfskehl im Mittelmeer-Zyklus stehen. Manchmal offen, manchmal verdeckt, trägt und strukturiert dieser Gedanke den gesamten Zyklus. Mit der Vorstellung, er, Wolfskehl, sei der „Ultimus Vatum“, stellt sich das abschließende, "Das Fünfte Fenster" an das Ende dieser Tradition.

Schon mit dem „Vorspruch“", und dort schon in der ersten Zeile, wird auf George verwiesen: "Ewe" ist ein von George im Deutschen geschaffenes Wort, gebildet nach dem mittelniederländischen "êwe", niederländisch eeuw, für "Zeitalter", "Jahrhundert". Mit dieser Bedeutung erscheint es in Georges „Jahrhundertspruch“:
Zehntausend sterben ohne klang: der Gründer
Nur gibt den namen. Für zehntausend münder
Hält einer nur das maass. In jeder ewe
Ist nur ein gott und einer nur sein künder. ${ }^{6}$

\begin{abstract}
Als diesen einen Künder sieht sich nun Wolfskehl. Bevor dies weiter entwickelt wird und die Frage beantwortet werden soll, wer denn der Gott ist, von dem er künden will, einige Erläuterungen zu dem rätselhaften „Vorspruch“. Die äußerste Verknappung der ersten Zeile ist doch wohl als Anruf zu lesen: "Ihr Dulder, die ihr mit eurem Leid dem Zeitalter, in dem ihr gelebt habt, seine Weihe gegeben habt", - mit der implizierten Bitte des Musenanrufs: "Gebt mir die

5 Ebd., S. 455.

6 Ebd., S. 338.
\end{abstract}


Kraft, daß ich mit meinem aus meinem Leid geborenen Gesang, auch meinem Zeitalter seine Weihe gebe". Auch "Weihe" ist ein George-Wort. - Es folgt zunächst eine Gruppe von vier "Duldern": Prometheus, der den Göttern das Feuer stahl und dafür an einen Felsen gekettet von einem Geier sich die Leber fressen lassen mußte, Herakles, der sich auf einem Holzstoß verbrennen ließ, weil er die Schmerzen nicht mehr ertragen konnte, mit denen ihn das Nessos-Hemd zerfraß, Hiob, der im Staube zu Gott schrie, und Christus am Kreuz. Dann aber folgt, wie es scheint, ein Fünfter, Dionysos, der aber "Fünfter" nicht genannt wird. Als "Fünften" Dulder führt Wolfskehl nämlich dann sich selber ein: "Fünfter Du, Enteiler und Verweiler". "Enteiler", weil er zwar in corpore ins ferne Exil gegangen ist, als "Verweiler" jedoch in spiritu in der vom Mittelmeerischen geprägten europäischen Welt beheimatet bleibt. So wären es eigentlich sechs Figuren, von denen hier die Rede ist. Wolfskehl geheime Rechnung sieht aber anders aus: Dionysos, der von den Mänaden zur "Wendefeier" der Wintersonnenwende "zerstückt", in "Werdeschauern" immer wieder neu geboren wird und Fruchtbarkeit und neues Leben bringt ("Vergeher", "Gedeiher"), kann außerhalb der Zahlenreihe bleiben. In diesen "Werdeschauern" überströmt, 'überschäumt' er nämlich die vier vorangeggangenen Dulder, "Mittmeers Weiher", nimmt sie in sich auf, so daß aus Vier und Einem Vier in Einem werden. Gleichzeitig, in einer zweiten Bedeutung des Wortes "Weiher", überströmt und "überschäumt" Dionysos auch die Grenzen des Mittelmeers "Weiher" nun gesehen als "kleiner Teich", um den die klassische Welt gelagert war. Er wird als einziger "Gott" genannt, wie in Georges „Jahrhundertspruch" "nur ein gott" ist, und "sein künder" will Wolfskehl sein.

An der vermeintlichen Fünfzahl des Vorspruchs stieß sich die klassische Philologin Renata von Scheliha, der Wolfskehl den fertigen Zyklus geschickt hatte: "Drei und vier sind heilige Zahlen, fünf aber ist die Zahl, die in jedem attischen Monat dies nefas war, [...] Und fünf ist die Zahl der Erynnien." (BaN II, S. 824) Wolfskehl antwortete sehr erregt:

Das was Ihnen mehr beiläufig in die mahnende Feder fließt, dem Dichter ist es [...] strukturell von unmittelbarer Bedeutung. [...] Eben diese kruziale Fünf ist nämlich die tragende Grundzahl dieses Zyklos, und da sie die Grundzahl ist, dringt sie vor, oder schimmert durch, bis in alle Einzelheiten des ganzen Gebildes. Ich erkläre auch nicht, was dem Dichter die Fünf als inneres Erlebnis hier bedeutet, 
vielleicht noch mehr als bedeutet. Prüfen Sie nach, [...] wie und wieweit hilfreiche oder mitspielende Neben- und Sonderzahlen zur Fünf stehen in dieser Dichtung. [...] Über die allerdings ungeheure Wichtigkeit einer Wandlung der Pemptas in die Hebdomas im apollinischen Bezirk schweigt man besser selbst mit Mysten. Nur rufe ich ins Gedächtnis, daß des größten Julius Geburtsstern in der selben Gleichung steht, und daß der letzte Monat, obwohl der Numeration nach der zwölfte, heute noch davon der Zehnte genannt wird. [...]

[...] Der "Vorspruch" [...] kündet auch heute noch nicht von Fünf Duldern sondern, was im Geheimverstand ganz und gar nicht das selbe ist, von Vier und Einem (Warum der Eine zugleich der Fünfte genannt wird und doch an erster Stelle steht in der End-Apologie), lege ich nicht aus. Diese Vier aber, und das ist nicht ihre 'Bedeutung', denn sie sind keine Allegorien, sondern es ist ihr Sein, überwinden durch die Dulden [sic!] in der geheimnisvollen Zahl und Richtung, daraus das Universum für unsere Art, auch also und sonderlich für Unser Meer (die "Erdmutter") sich auferbaut. Und also stellen sich die vier Elemente dar, als Bewegung gefaßt: der Dulder am Fels ist Überwindung von Außen. Das meint das Titanische. Der Dulder am Stoß ist Überwindung von Innen. Das ist "der mann, der immer geist ist" [Anm. - abgewandeltes Zitat aus Stefan George, Stern des Bundes: Die weltzeit die wir kennen schuf der Geist / Der immer mann ist..."] Der Dulder im Kot ist die Überwindung von Unten. Denn der Knecht tut den ersten Schritt und da mit den der Entscheidung zu eben jenem Gott, der im Dulder am Kreuz seinen Sohn herabläßt als Überwindung von Oben, als "Fleisch gewordenen "Gute Botschaft". (BaN II, S. 826 f.)

Was mit der "Überwindung von Außen", "von Innen", "von Oben" und "von Unten" gemeint ist, kann hier nicht erläutert werden. Auch von Wolfskehl wird dies im Mittelmeerzyklus nicht weiter entwickelt, wohl aber in den Zyklen INRI und Hiob, zu denen sich Paul Hoffmann und Gunter Grimm geäußert haben. Hier geht es um das seltsame Zahlenspiel. Wolfskehl verdeutlicht dann nämlich, warum die Vier und Einer nicht dasselbe sind wie die vermeintlichen Fünf, daß nämlich Dionysos, "der 'Gott Befreier', Er der 'Lebensschauer', Er der 'Todestraum' so wie Er Mittmeers Weiher 'über-schäumt', also auch, die Vier Meiler in sich fassend überwölbt" und fügt hinzu: “(s. 'Maskenzug', Auslese III, p. 55, V. 10: 'Denn euer keinen miss ich in dem Kreis')" (BaN II, 828). Als "Alldionysos" fasse er "alle Keimung in sich" - implicite also auch die vier erstgenannten "Dulder" und letztlich ihn, Wolfskehl, selbst, der im „Vorspruch“ dann als „Fünfter“ mit dem Unge- 
zählten verschmilzt: "Fünfter Du, Enteiler und Verweiler". Die Nähe zu Dionysos wird in der letzten Strophe des Zyklus noch einmal hergestellt:

Denn wen Wandlung umschuf zu Kür und Sende,

Gärt, Evios, ewig aus Allgottes Lende, [...]

Eine doppelte "Sohnschaft" wird so beschworen: "Kür und Sende" etablieren, durch "das Wunder der Verwandlung", die Vaterschaft Georges, "Allgottes Lende" etabliert diejenige von Dionysos.

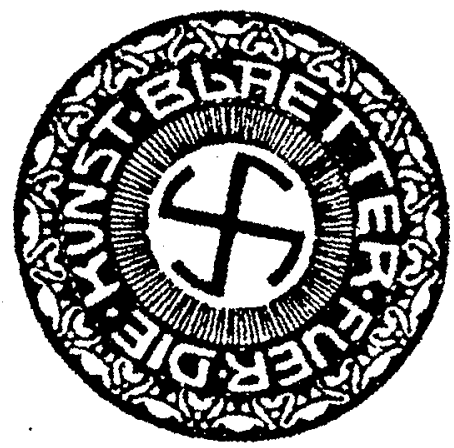

Die komplizierte Denkfigur und das geheimnisvolle Zahlenspiel mit der "kruzialen Fünf" als "tragender Grundzahl des Zyklos", wie es im Brief an Renata von Scheliha hieß, findet eine verblüffende Lösung: Zunächst einmal läßt "die kruziale Fünf" an das Signet denken, das Melchior Lechter für Georges Blätter für die Kunst entworfen hatte: ein Swastika-Symbol, das man auch als zwei überkreuz liegende Fünfen ansehen kann. Für diese Zeitschrift, die nur im George-Kreis zirkulierte, hatte Wolfskehl den Begriff des "Geheimen Deutschland" geprägt, das nur dort vertreten sei. Weitere Andeutungen finden sich im selben Brief. Ausdrücklich betont Wolfskehl dort, daß er dem Leser keine "gematrische", also kabbalistisch-zahlenmystische "Schlüsselaufgabe" stellt. Vielmehr verweist er auf den Maskenzug und auf "des größten Julius Geburtsstern". Sowohl Wolfskehls "Dramatische Dichtung" Maskenzug von 1904 als auch Georges gleichnamiges Gedicht beziehen sich auf den Maskenzug, der am 22. 2. 1903 in Wolfskehls Haus stattfand. Hier trat Wolfskehl als Dionysos auf. Wir wissen, in welch hohem Maße Wolfskehl immer wieder das Dionysische in seiner Person und seiner Lebenswelt ausgeprägt sah und wie "dio- 
nysisch" er von anderen empfunden wurde. Ausdrücklich bezieht er sich im Mittelmeer-Zyklus auf seine Dionysos-Verkörperung beim Maskenzug von 1903, wenn er in den aus dem "Fünften Fenster" zitierten Zeilen George sagen läßt: "Die Tracht und Pracht von damals bringst du mit./ Entbürde, gürte dich zum Tänzerschritt". Für das Zahlenspiel des Vorspruch zum MittelmeerZyklus kann das nur heißen, daß Wolfskehl, von George dazu aufgefordert, wiederum in die "Tracht und Pracht von damals" schlüpft, mit Dionysos verschmilzt, der nun seinerseits als "Allgott" die vier erstgenannten "Dulder" Prometheus, Herakles, Hiob und Christus in sich aufnimmt, so daß - wie Wolfskehl im Brief schreibt - nicht von fünf "Duldern" gesprochen wird, und schon gar nicht von insgesamt sechs Figuren, wie es scheinen könnte, sondern von "Vier und Einem". Das ist die eine Bedeutung der "kruzialen Fünf", der Wolfskehl im Brief so eine große Bedeutsamkeit zumißt. Es gibt aber noch eine zweite.

Beim selben Maskenzug war George als Julius Cäsar aufgetreten (sowie Henry von Heiseler als Hermes und Alfred Schuler als Magna Mater). Auf Cäsar weist Wolfskehl im Brief an Renata von Scheliha ausdrücklich in Verbindung mit der Fünfzahl in "Mare Nostrum" hin: "Nur rufe ich ins Gedächtnis, daß des größten Julius Geburtsstern in derselben Gleichung steht". Nun wurde Julius Caesar, ebenso wie George, an einem 12. Juli geboren, "des größten Julius Geburtsstern" ist also auch der Georges. Für Wolfskehl in seiner George-Verehrung, und auch wohl für George in seinem Selbstverständnis, das ihn im Maskenzug die Cäsarmaske wählen ließ, konnte dies kein Zufall sein, vielmehr ist es, wie Wolfskehl in dem genannten Brief hinzufügt, "ein 'kosmischer'Akt des Divus in Person". Nun war im altrömischen Kalender der Juli der fünfte Monat ("Quintilius"), und der Dezember, wie Wolfskehl ebenfalls angibt, der Zehnte, im Dezember aber starb George. Caesar selbst führte die neue Zeitrechnung mit einem neuen Kalender ein, der nach ihm der Julianische genannt wird. Ebenso begann, für den George-Kreis eine neue Zeitrechnung mit dem "Meister". Die Fünf steht also auch für George und für den Abschluß einer alten und den Anbruch einer neuen Zeit: "Zu neuem Reich gefeit an Neuer Wende", heißt es wiederum in der letzten Strophe des Zyklus. Es scheint, als übernehme Wolfskehl auch damit eine Technik Georges. Denn für die Zeichenhaftigkeit, die Wolfskehl hier mit der Fünfzahl entwickelt, hatte ihm "der Meister" schon mit der Sieben ein Vorbild gegeben: In Georges Der Siebente Ring gibt es sieben 
Bücher mit einer jeweils durch sieben teilbaren Anzahl von Versen. Machen wir einige Stichproben, wie, in Wolfskehls Worten, die Fünf als "tragende Grundzahl"."bis in alle Einzelheiten des ganzen Gebildes" 'vordringt' und 'durchschimmert' mit 'hilfreichen Neben- und Sonderzahlen'. Der "Vorspruch" hat 20 Zeilen, teilbar durch zehn, durch fünf, aber auch durch vier, so daß eine arithmetische Gleichung von "vier und Einem" "Dulder" entsteht. Wolfskehl verweist in dem Brief an Landmann und Scheliha im Bezug auf die Fünfzahl auch auf "das Geheimnis der Perspektive". Sie ergibt sich aus den durch vier Punkte erstellten Fluchtlinien, die auf einen fünften Punkt, den Fluchtpunkt zulaufen. Dieser Fluchtpunkt liegt zumeist in der Mitte. Anders anschaulich gemacht, könnte man an einen Tafelaltar denken, mit einem Zentralbild und jeweils zwei Tafeln an den Seiten. Ein solches Zentralbild ist im Mittelmeer-Zyklus ohne Zweifel das bei weitem umfangreichste, das Dritte Fenster mit dem Titel „TABELLAE“. Der Titel verweist auf Georges "Tafeln" aus dem Siebenten Ring, ebenfalls zumeist fünfhebige Vierzeiler, von denen Wolfskehl dann im "Fünften Fenster"einzelne direkt aufruft. Es sind 41 "tabellae", eine Zahl, auf die Wolfskehl großen Wert legte; auch "Der Vierte Spiegel" von Hiob oder die Vier Spiegel hat 41 Verse. Gunter Grimm, in seiner Untersuchung zur Hiob-Dichtung, verweist darauf, daß es sich bei der 41 um eine der von Wolfskehl besonders geschätzten, da in sich perfekt-unteilbaren, Primzahlen handelt, deren Quersumme wiederum fünf ergibt.7 Auch sieht Grimm darin ein Bezugnehmen auf Wolfskehls Leben: "Die persönliche Bekanntschaft zwischen Karl Wolfskehl und Stefan George begann im Jahre 1893 und dauerte bis zum Tode im Jahre 1933. Das sind exakt 41 Jahre." In der fünften Strophe des "Fünften Fensters" erfolgt der hymnische Preis der Offenbarung, die ihm in dem berühmten "Kugelzimmer" zuteil wurde, das er George in seiner Münchner Wohnung nach dessen Wünschen eingerichtet hatte:

O welch Verstummen, als im Kugelzimmer

Ver Sacrum aufging, Andrer Sphäre Schimmer

Die Kränze rührte [...]

Und wir erinnern uns: es war die abschließende, die 41. Tafel der „Tabellae", mit der Quersumme fünf, wo Wolfskehl von "den Er-

7 Grimm, Gunter, Karl Wolfskehl. Die Hiob-Dichtung, Bonn 1972, S. 213214. 
lauchten" spricht, "die unser Meister sich befahl zum Heer,/Zur Schutzwacht Neuem Reiche Mittelmeer." Schließlich wird George in der 25sten Tafel der Tabellae - das ist fünf mal fünf - mit seiner düstersten Untergangsvision aus "Die tote Stadt" zitiert, wenn es heißt:

\footnotetext{
Der Mensch vergeht. Schon brüllt formlose Masse Nach Zwängerwahn gepresst, und rühmt sich Rasse?

"Schon eure Zahl ist Frevel" - droht der Meister, Und fürchterlichsten Untergang verheisst er.
}

(Wir können die unfreiwillige Komik dessen, was Gunter Grimm Wolfskehls "Wilhelm-Busch-Reime" im Mittelmeer-Zyklus nennt, freundlich ignorieren, wir können sie aber auch als Beleg dafür nehmen, wie sehr gerade hier Anspruch und Mittel auf fast groteske Weise auseinanderklaffen.) In Georges „Die tote Stadt" gilt die Verheißung des 'fürchterlichsten Untergangs' einer dem Materiellen verfallenen und daher verworfenen "Masse", die den wenigen Auserwählten gegenübergestellt wird:

Das gut was euch vor allem galt ist schutt.

Nur sieben sind gerettet die einst kamen

Und denen unsre kinder zugelächelt.

Euch all trifft tod. Schon eure zahl ist frevel. 8

Gunter Grimm macht die "kruziale Fünf" noch auf andere Weise sichtbar. Er zählt zum Beispiel im "Fünften Fenster”, „Ultimus Vatum", die Anzahl der Verse in den insgesamt 17 Strophen und kommt auf folgendes Ergebnis:

$6--4-4-4-4-4-5-5-6-4--6-5-5-4-4-4-4-4--6$

Deutlich erkennbar ist, was Grimm die axial-symmetrische Anordnung nennt: Um eine zentrale neunte Strophe mit vier Versen sind spiegelbildlich zwei Fünfergruppen angelegt, die ihrerseits wieder gerahmt sind von je zwei sechsversigen Strophen, die jeweils mit der vier in der Mitte eine Zehn ergeben. Die zentrale neunte Strophe zitiert ein George-Wort - "Blutschmach" - aus Der Krieg, wo es heißt „Wollt uns bewahren vor zu leichtem schlusse / Und vor der Blutschmach! Stämme / Die sie begehn sind wahllos auszurotten“ Wolfskehl münzt das auf den Nationalsozialismus und seinen Blutund Bodenkult um: "Ich sah die Schmach, die Blutschmach, Bodenschmach". Warum gerade dies für Wolfskehl eine überragende

8 George, Werke, S. 244. 
Bedeutung hatte, soll gleich erklärt werden.

Neben der Fünf und der Vier spielt auch die Sieben im MittelmeerZyklus eine bedeutende Rolle. "Nur sieben sind gerettet" hieß es in Georges "Die tote Stadt" aus dem Siebenten Ring. - Kein Zufall wird es sein, daß die Sieben, für Wolfskehl durch Georges Siebenten Ring noch zusätzlich geheiligt, auch die Zahl ist, die hinter dem "Zweiten Fenster", der „Appellatio“, steht: In sieben Strophen wird der "Wahlheimat" gedacht, jenes Italiens von Wolfskehls erster Zuflucht, die eben noch nicht als Exil, sondern als Urheimat, als "Mittelmeeres Mutter" empfunden wurde. Dort in Camogli bei Recco war er 1914 schon zusammen mit George gewesen. George hatte dort die drei Gesänge „An die Kinder des Meeres" geschrieben und Wolfskehl Gedichte wie "Die Kelter", „Die Kuppe“ und „Die Bucht“. In den „Kindern des Meeres“ scheint noch einmal das Motiv der "Sohnschaft" auf, und Wolfskehl wird im Rückblick Georges Worte aus dem dritten der Gesänge als prophetisch auf seine Reise ins ferne Neuseeland bezogen haben:
Versippter uns durch der Gemeinschaft brauch
Wirst bald du fahren - unserer hut entzogen -
Macht-rühmlicher! Aus deinem edlen hafen
In welches neue land auf welch ein meer??

Oder aus dem ersten Gesang die Zeilen:

Seefahrend heil und sucht des abenteuers reisst dich - den heftigen zauber frommer tage Aus unseren augen auf das fernste meer. 10

Denn Wolfskehl antwortet im "Abgesang" des Großzyklus, Georges Worte wieder aufnehmend, daß "der Ruf mich fernhin reisst" und stellt das George-Zitat voran: "Nur aus dem fernsten kommt die erneuung".

Auch in Wolfskehls Camogli-Gedichten, wie in dem berühmten „Die Kelter“, wird das Meer beschworen oder, wie Rudolf Pannwitz dazu schreibt, "das Zusammensein mit George, die Realvision von Brunnen, Kuppe, Bucht, Meister: in Horizonten gefangene und entfaltete Segensfülle des Dionysos-Apollon":

9 Ebd., S. 408.

10 Ebd., S. 406. 
Grad ob dem meer stand ich, seine haut

Weit ausgespannt zerrieb sich hell am himmel

Und sein gepreßter Atem keuchte laut,

Doch lauter schrie die stimme

Die über wolken zu mir flog

mit adlerprall und zornigem gerüttel

Die Pforten aufbrach und die seelen sog:

Ihr herzen in die bütte!

Trauben in eigner flamme gar gekocht

Der Winzer ist erwacht, der erntebrüller

Fährt in den wingert, euer saft erpocht

Zersprengt die hülle.

Ich will euch stampfen hochgeschürzten beins

Quellt über, gischt im kelternden gewitter,

Ertränkt und schwängert heilige fluten weins

Mich heilige Mitte!

Dies alles ist mitzudenken, wenn Wolfskehl im "Zweiten Fenster", in der Siebenzahl der "Appellatio", die Wahlheimat Italien und damit das Mittelmeer anruft. Wieder wird im Wein das Dionysische, in dem sich beide Dichter und beider Gedichte verbunden gefunden hatten, evoziert, um so - nicht unähnlich dem "Heiligtrunkenen” in Hölderlins „Brod und Wein“ - den Sprecher zu erheben in den Flug der dichterischen Phantasie:

Wahlheimat, unser hellstes, unser freistes

Festlichstes Lachen klang wenn wir im Schein

Der Frühe dir zujauchzten, wir, du weisst es,

Aus tausend Bechern tranken deinen Wein.

Wahlheimat, wo im Licht auf Griechischem Hügel

Bei Vorgebirgs delphinisch reinem Bug

Der Meister stand, umrauscht vom Schwanenflügel -

O welche Frucht der griechische Hügel trug!

(Zum "griechischen Hügel schreibt Margot Ruben in ihren Erinnerungen: "Unser schönster Weg zum 'Griechischen Hügel', den George und Karl Wolfskehl damals täglich erstiegen."11) Als Lie-

11 Ruben, Margot, "Karl Wolfskehl. Gespräche und Aufzeichnungen. 19341938". In: Karl Wolfskehl, Kalon Bekawod Namir. "Aus Schmach wird Ehr". Amsterdam 1960, S. 97. 
bender, Einlaß erbittend, naht er sich dann im Geiste dem Mittelmeer in der letzten, der siebenten Strophe, um es als Orakel zu befragen:

Den Spruch, wie er auch gehe, hör die Schwüre,

Ich nehm ihn an, ich der dir einst genaht,

Ein Liebender, beschenkt, dankvoll: die Türe

Sperrst du sie, öffnest du sie, Wahlheimat?

Die Erhörung dieser Bitte um Einlaß und der Spruch des Orakels machen nun die verbleibenden drei Teile des Zyklus aus: Als zentraler und umfangreichster Teil steht, wie schon gesagt, das "Dritte Fenster: Tabellae" noch einmal ganz im Zeichen Georges. Mit seinem Titel und seinen 41 fünfhebigen Vierzeilern verweist es auf Georges ebenfalls fünfhebige und zumeist vierzeilige "Tafeln". Woher diese zentrale Position rührt, dürfte nun deutlich geworden sein: Schon die zweite von Georges Tafeln hatte sich "An Karl und Hanna" Wolfskehl gewandt.

In einem gewaltigen Panorama wird der Bogen geschlagen von den mythischen Anfängen des Mittelmeeres in der ersten Tafel, "da der Halbgott dir die Sperre brach" - das heißt die Säulen des Herkules den Atlantik in das Mittelmeerbecken einströmen ließen - bis zu der Gegenwart Wolfskehls in Neuseeland in der letzten, der 41. Tafel, in der Wolfskehl sich "Zur Schutzmacht Neuem Reiche Mittelmeer" berufen sieht. Daß schon die zweite Tafel, wie bei George Wolfskehl, so bei Wolfskehl Dionysos einführt, ist eine sicherlich bewußt vorgenommene Parallel-Plazierung, um die schon im „Vorspruch" vollzogene Verschmelzung Wolfskehls mit der DionysosMaske noch einmal subtil zu unterstreichen.

Zwischen Anfang und Ende des "Dritten Fensters" entfalten sich Mythos, Geschichte und Kultur der mittelmeerischen Welt im Zeichen des Verrats durch den Ungeist von Faschismus und Nationalsozialismus: "Schnürt antikisch eure Marter-Ruten,/Steckt drein die Metzelaxt: o tot Symbol!" - das ist das Fasces-Bündel Mussolinis, das sich exakt in der Mitte, in der XXI. Tafel, erhebt. Dagegengehalten werden die Großen der Antike, des Mittelalters und der Neuzeit: Homer, Caesar, Sankt Peter, aber auch Napoleon, Vergil und Dante, schließlich Platon und - da fällt es schwer, den in der Fast-Homonymie gegebenen Sprung nachzuvollziehen - unmittelbar danach Platen, Graf August von Platen-Hallermünde, der als Verfemter im italienischen Exil wohl an Wolfskehls eigenes Schick- 
sal gemahnte. - Hier bedürfte es eines umfangreichen Kommentars und einer ausführlichen Interpretation, die in diesem Rahmen nicht geleistet werden können.

Noch mehr gilt das für die beiden letzten Teile seines Zyklus, „Vaticinium" und „Ultimus Vatum“, in denen er nach eigenem Selbstverständnis ganz als "Vates" spricht: "Hier spreche ich nicht ex cathedra sondern ab altare", wie er es in dem eingangs zitierten Brief an Renata von Scheliha betonte. Wenn er sich hier derart, wie in dem früheren Ausspruch "Der Dichter ist die Durchbruchstelle für die Wirklichkeit der Götter"12 als Wissenden und Künder nicht stilisiert sondern tatsächlich glaubt, werden wir ihm nicht mehr folgen wollen. $\mathrm{Zu}$ anachronistisch, weltfremd und konstruiert wirkt im „Vaticinium“ die Erhebung zu Schicksalsnornen der drei ihre Söhne beklagenden Mütter: Cornelia, Mutter der Gracchen, Rustikiana, der Witwe des Boethius, die nach dem Goteneinfall in Rom zur Bettlerin wurde, und Laetizia, der Mutter Napoleons, deren Warnung "Pourrvou que ça dour!" (= "Wenn das nur Bestand hat!") den basso continuo der Untergangsvisionen für das "Vierte Fenster" abgibt. Gewiß, in jedem Fall ließen sich die in den drei Müttern personifizierten Umbruchssituationen als Parallelen zu dem Unheilsschicksal ziehen, das Wolfskehls Gegenwart bedrückte: der Tod der sozialen Gerechtigkeit nach der Ermordung der Gracchen durch eine habgierige Herrenklasse, die Barbarei, die nach dem Gotensieg über die römische Welt hereinbrach ("Rustikiana empfand ich von je als die wahrhaft symbolische Gestalt in der Agonie und Metamorphose der antiken Urbs", schreibt Wolfskehl einmal dazu), und schließlich Napoleon, der "der Crapule" erlag, wie es im Vaticinium heißt, so wie dann die abendländische Kultur der "Brut der Kerfe", also des nazistischen Ungeziefers. Im übrigen bestand im George-Kreis eine ausgesprochene Verehrung für Napoleon, wie auch eine Bemerkung Wolfskehls bezeugt: "Mit Napoleon tritt das Menschentum in einen neuen Werdenskreis, einen neuen Eros [...] Er und seine Paladine sind zugleich die wiederkehrende Artus- oder Karlsrunde der christrömischen Welt und als Erlöser und Zerstörer des gespenstisch gewordenen alten Europa, Heiland und Sendbote dessen, was kommt."13

Im letzten, im "Fünften Fenster" findet nun, unter dem Blickwinkel des Exils, die Engführung aller Motive statt, auf die der Zyklus hinstrebt. Schon im Titel „Ultimus Vatum" tritt Wolfskehl die Nachfolge Georges als Seher an, stellt dann die Rückbindung

12 Ebd., S. 129.

13 “De Coster und der Ulenspiegel”, GW II, S. 322. 
her an die Ahnen, die aus der Toskana durch Karl den Großen an den Rhein gerufen wurden - so Mittelmeer und Deutschland verbindend - und läßt sich von George zur Dionysos-Verkörperung küren: "Die Tracht und Pracht von damals bringst du mit./Entbürde, gürte dich zum Tänzerschritt." Unmittelbar, wie auch in der auf Hölderlins „Brod und Wein“ verweisenden „Appellatio“ des "Zweiten Fensters", geht aus der Ergriffenheit durch den Weingott das dichterische Wort hervor, noch einmal das frühe CamogliGedicht „Die Kelter“ beschwörend:

Exul! Mich Keltrer, Kellerer des Weins,

Des Weins, der Wort heisst, Geist, Tau des Gedeihens,

Mich den bestellten Hüter Unsres Schreins,

Mich wies ein Wicht in Acht und Aberacht,

Griff mir ans Herz und trieb mich in die Nacht.

Erst aus der Gesamtschau des Zyklus kann man nun verstehen, warum ein in der Geschichte der vierziger Jahre scheinbar so nebensächliches Ereignis wie das Hitler-Mussolini Abkommen von 1939 über die Aussiedlung der deutschen Bevölkerung in Südtirol "Heim ins Reich" ein Schockerlebnis für Wolfskehl war, das die zweite Hälfte von „Ultimus Vatum“, und damit den Höhepunkt des Zyklus, bestimmt. In einem Brief an Salin vom 7. 8. 1939 hatte Wolfskehl dazu geschrieben:

Und wie bitter mundet nun auch der Heimwehtrank nach der letzten fürchterlichen Geschichtsklitterung. Dietrich von Bern endgültig aus seinem geheimsten Bezirk gejagt, die Herrin Virginal in ihre innersten Klüfte niedergescheucht, der Rosengarten zertrampelt, der Hain vor unseren Toren zersägt und entwurzelt. Ich hätte nicht geglaubt, daß mich noch irgendetwas so weh schütteln könne bis ins erschauernde Mark. Und wie dacht ich unseres Zusammenseins vor zwei Jahren und Wolfram von Steinens Frühlingsfahrten in jenes deutsche Land, dessen Hauptstadt bis heute "Ostermünchen" [d. i. Bozen] heißt. Und Fallmerayer, edelster Sprecher in der Runde des geheimen 19. Jahrhunderts, war nahe bei Brixen zuhaus. (BaN II, S.132)

Die Schlußverse des Zyklus werden, bis in wörtliche Anklänge und gedankliche Übernahmen, vorgegeben durch die Zeilen, die Wolfram von Steinen im Juli 1939 zum selben Ereignis an Wolfskehl schreibt: 
[...] was wäre BLUTSCHMACH wenn nicht dies, [...] was jetzt ansetzen soll: die landschaft Dietrich von Berns und Walther von der Vogelweides, mit einer stadt der erst vor einem menschenalter der Meister eine tafel gesetzt hat [„Bozen. Erwins Schatten“, in: Der Siebente Ring, bei Wolfskehl "Bozen, drin Erwins Spuren"], dies vielgeliebte blütenland nicht nur verloren zu geben sondern zur ausrottung ("aussiedlung") ihres alten immer treuen stammes selber die hand zu bieten [...] Wenn dennoch einmal deutsche siegen werden so werdens andere deutsche sein - das wahre volk - jenes beste gut das zum banne ging...

Es muß sehr vieles, unheimlich vieles zerfallen, sehr vieles zer schlagen werden, sehr vieles geprüft und durchgerüttelt und gewezt werden bevor ein neuer tag erscheinen kann, ein göttertag. Und je deutlicher dieses stets geahnte und schematisch gewußte muß vors geistige auge tritt und ereignis wird, um so unfaßlicher erscheint das wunder das Sie, Karl Wolfskehl, erlebten und heute allein bezeugen, die kunft eines boten von diesem tag, die wende der zeit. (BaN II, S. 306 f.)

Hier war also die Brücke zerstört, die Deutsches und Mittelmeerisches verbunden hatte, die von Rom herrührende Reichs- und Kaiseridee zerbrochen, die in Wolfskehls Verständnis seiner selbst und seiner Sippe eine so bedeutende Rolle gespielt hatte ("den Kaisern zugehörig" wie es zu Anfang des "Fünften Fensters" heißt), und es war die Heiligung, die die Landschaft durch den George-Kreis erfahren hatte, besudelt. Für den George-Kreis war Südtirol nämlich das Eingangstor zu jenem mythischem StauferReich, für das George Claus Schenk Graf von Stauffenberg reklamieren wollte, da dessen Name in direkter Linie von den Staufern abstamme. Hier bewegen wir uns also im Sanktuarium des "Geheimen Deutschland". Ruth Bowert schreibt zu diesen Versen und dem sie verteidigenden Brief Wolfskehls an Viëtor, der von Viëtor beanstandete Nationalismus Wolfskehls gründe sich "auf einer erlebnismäßig angeeigneten Geschichtsauffassung, die sich die Wiedererstehung eines staufischen Großreichs erträumt, dessen kulturelle und politische Schwerpunkte dann Österreich, das Ultramontane und der Rhein wären. In diesen Gebieten habe sich für den Deutschen das Mediterrane in Herrschern und dichterischen Einzelgestalten realisiert."14 Jüngst hat noch einmal Gustav Seibt

14 Bowert, Ruth, Die Prosa Karl Wolfskehls. Grundzüge seines Denkens und seiner Ausdrucksformen. Hamburg 1965, S. 269. 
darauf hingewiesen, welch großen Einfluß in diesem Zusammenhang das 1927 erschienene Staufer-Buch von Ernst Kantorowicz, Kaiser Friedrich der Zweite, auf den George-Kreis hatte, besonders da es sich im Vorspruch "im Geiste des Dichters Stefan George" ausdrücklich berief ,,auf ein 'geheimes Deutschland', auf die Kaiser und Helden einer besseren Vorzeit 'gerade in unkaiserlicher Zeit'".15 In einem Brief an George vom 26. 11. 1933 hatte Kantorowicz dann, im Hinblick auf das Interregnum von 1250 bis 1273, "die notwendige geburt eines 'geheimen Deutschland' nach dem zusammenbruch des stauferreiches" beschworen: "Denn die staufer hatten ja - zum einzigen mal in der deutschen Geschichte das 'geheime Deutschland' von damals i. e das 'RÖMISCHE' zum offiziellen Deutschland erhoben..." So wie damals könne das 'Geheime Deutschland' auch heute nur ein geistiges sein, "gelenkt von seinen Kaisern und einem geistigen Adel". 16 Wolfskehl macht deutlich, daß er im „Ultimus Vatum" an das "Geheime Deutschland" als geistiges Kontinuum denkt, wenn er im Gedicht und in dem zitierten Brief an Salin auf den Südtiroler Historiker Jakob Philipp Fallmerayer als auf den "edelste[n] Sprecher in der Runde des geheimen 19. Jahrhunderts" verweist. Es ist die durch die Aussiedlung der Deutschstämmigen aus Südtirol bedrohte Geographie eines solchen mythisch-geistigen Kaiserreichs, die Wolfskehl in Strophe 11 in die Worte faßt: "Den Kaisersaum kappt ihr vom Reichsgewande".

Hierin also liegt die zentrale Bedeutung, die Wolfskehl der ebenso zentral gesetzten neunten Strophe verleiht, wenn er sie axial-symmetrisch als Fluchtpunkt für das "Fünfte Fenster" wählt, - noch einmal das Prinzip der Perspektive anwendend, von dessen "Geheimnis" in bezug auf die Fünfzahl Wolfskehl in dem genannten Brief an Landmann und Scheliha gesprochen hatte:

Ich sah die Schmach, die Blutschmach, Bodenschmach.

Nicht wie Despotenfaust sonst Äste brach,

Den Stamm beliess: samt seinem Mutterstollen

Verfeilscht der Forst, draus lang uns Sang erschollen.

Mit dem George-Zitat von der "Blutschmach" in dieser neunten

15 Gustav Seibt, "Der Staat als Kunstwerk. Das geheime Deutschland und der Widerstand." In: Ders., Das Komma in der Erdnußbutter. Essays zur Literatur und literarischen Kritik, Frankfurt a. M., Hamburg 1998, S. 39.

16 Zitiert nach: Hoffmann, Peter, Claus Schenk von Stauffenberg und seine Brüder, Stuttgart 1992, S. 115. 
Strophe, das ihm in dem Brief von Wolfram von den Steinen zugereicht worden war als Aufforderung, einen 'neuen tag', 'einen göttertag zu beschwören und "allein [zu] bezeugen, die kunft eines boten von diesem tag, die wende der zeit", war der Anruf aus dem George-Kreis an ihn ergangen, die Vision vom "Geheimen Deutschland" zu bewahren. Auf diese Vision läuft der Mittelmeerzyklus bezeichnenderweise in seinem "Fünften Fenster" hinaus. Aus diesem Anruf und aus dieser Vision bezog Wolfskehl denn auch seinen hyperbolischen Anspruch, mit der "sohnschaft" Georges auch dessen Seherrolle zu übernehmen. - Der "göttertag" aber, von dem Wolfram von den Steinen spricht, ist der des Gottes der zyklischen Erneuerung in der ewigen Wiederkehr, des Wendegottes, des mittelmeerischen Dionysos, mit dem der Zyklus in der Hoffnung auf das Kommen eines "Neuen Reiches" schließt. In seiner durch den Maskenzug von 1903 begründeten Verkörperung dieses Gottes nimmt Wolfskehl dessen Ankunft vorweg:

Denn wen Wandlung umschuf zur Kür und Sende,

Gärt, Evios, ewig aus Allgottes Lende, $\mathrm{Zu}$ Neuem Reich gefeit an neuer Wende.

So taucht im Drang mystischer Wiederkehr Entbunden heim vom Rhein der letzte Seh'r, In letzter Sohnschaft heim in Unser Meer.

Er hatte mit "Mare Nostrum" "alles ausgesprochen, was mir über dies Ur-Mysterium der weissen Menschheit erlaubt und eröffnet ist". Die Frage bleibt, ob er damit nicht zuviel ausgesprochen hatte. Ist dem erschütternden menschlichen Dokument mit seinen hochartifiziellen privaten Zahlenspielen und George-Kreis-internen Verweisen noch ein Kunstcharakter abzugewinnen, der in Perfektion dem alles verschweigenden „Detail“ von Ursula Bethell und ihrer traditionellen aber subtil gehandhabten Zahlensymbolik vergleichbar wäre? 